\title{
Effectiveness of Cognitive-Behavioral Therapy and Amitriptyline in Patients with Chronic Temporomandibular Disorders - A Pilot Study
}

\author{
Patrícia dos Santos CALDERON ${ }^{1}$ \\ Maria de Lourdes Merighi TABAQUIM ${ }^{2}$ \\ Luiz Carlos de OLIVEIRA ${ }^{2}$ \\ Ana Paula Afonso CAMARGO ${ }^{2}$ \\ Tatiana de Cássia Ramos NETTO ${ }^{2}$ \\ Paulo César Rodrigues CONTI ${ }^{3}$ \\ ${ }^{1}$ Department of Dentistry, UFRN - Federal University of Rio Grande do Norte, Natal, RN, Brazil \\ ${ }^{2}$ Department of Psychology, USC - University of Sagrado Coração, Bauru, SP, Brazil \\ ${ }^{3}$ Department of Prosthodontics, Bauru Dental School, USP - University of São Paulo, Bauru, SP, Brazil
}

\begin{abstract}
The aim of this pilot study was to evaluate the effectiveness of cognitive-behavioral therapy (CBT) and use of amitriptyline, a tricyclic antidepressant, in patients with chronic temporomandibular disorders (TMD). Forty-seven women (mean age $=35.4$ years old) with chronic TMD were enrolled in the study and divided into 4 groups: amitriptyline; amitriptyline and CBT; placebo and CBT; and placebo only (control). Patients were managed for 7 consecutive weeks. Follow-up evaluations were done at the 1 st, 7 th and 11 th weeks of treatment. The presence and severity of pain, levels of depression, and quality of life and sleep were measured. Data were analyzed using ANOVA, Chi-square and Cochran tests, considering a significance level of 5\%. Improvements were found for all factors considered in the intragroup analysis, although no significant differences were detected among groups. However, at the end of the treatment (11 weeks of follow-up), these positive outcomes persisted only for the women treated with amitriptyline and CBT. The obtained results suggest that the combination of amitriptyline and CBT may be effective in reducing pain and depression levels as well as in improving the quality of life and sleep in patients with chronic TMD.
\end{abstract}

Key Words: antidepressant agents, behavioral sciences, temporomandibular joint disorders.

\section{INTRODUCTION}

Ongoing pain may be associated with co-morbid conditions such as anxiety, depression and sleeplessness. It is important to recognize and treat emotional distress as well as physical symptoms. Chronic pain and depression seem to share common neurochemical substrata and perhaps even similar dysfunctional alterations.

For the past several years, tricyclic antidepressants (TCAs) have been used successfully to manage and control a variety of chronic pain conditions. RizzatiBarbosa et al. (1) investigated a pharmacological protocol for the control of pain associated with chronic temporomandibular disorders (TMD) based on the use of amitriptyline and found that $25 \mathrm{mg} /$ day of amitriptyline was sufficient to significantly reduce the pain of chronic TMD without producing side effects.

Cognitive behavioral therapy (CBT) is also widely used in the management of these conditions (chronic pain and depression). The modalities of CBT vary in precise content but they have in common the following main principles: 1) the assumption that individual's feelings and behaviors are influenced by his/her thoughts; 2) the use of structured techniques to help individuals identifying and changing maladaptive thoughts and behaviors and 3) the emphasis on teaching skills that individuals can then apply more liberally when facing their problems.

The combination of CBT and TCA has not been reported in the treatment of TMD, and it is assumed

Correspondence: Profa. Dra. Patrícia dos Santos Calderon, Departamento de Odontologia, Universidade Federal do Rio Grande do Norte, Avenida Salgado Filho, 1787, Lagoa Nova, 59056-000 Natal, RN, Brasil. Tel: +55-84-3215-4111. Fax: +55-84-3215-4101. e-mail: patriciascalderon@yahoo.com.br 
that this combination would be advantageous because of the common co-morbidity between chronic TMD and some emotional conditions. Accordingly, the aim of this study was to evaluate the effectiveness of CBT and amitriptyline, in combination or alone, on the intensity of pain, levels of depression, and quality of life and sleep in patients with chronic TMD.

\section{MATERIAL AND METHODS}

\section{Subjects}

Participants were recruited from an outpatient University-based orofacial pain clinic at Bauru Dental School, USP, Brazil. Written informed consent was obtained for each patient according to the University Ethics Committee (Protocol \#101/2006).

Inclusion criteria were 1) history of orofacial pain for more than 6 months; 2 ) pain occurring daily or almost daily for at least the month preceding enrollment; 3) pain of at least moderate severity (i.e. at least $40 \mathrm{~mm}$ on a visual scale of 0-100 mm); and 4) age ranging from 17-55 years. Exclusion criteria included major neurological or psychiatric disorders, glaucoma, history of intolerance to amitriptyline, pain secondary to trigeminal neuralgia, or pain attributable to other local, well defined condition.

Initially, patients were classified as suffering from muscle-related and/or joint-related TMD, according to the guidelines of the Research Diagnostic Criteria (RDC) for TMD (2).

Among 400 patients evaluated, 60 were found to be eligible for the study and 47 were enrolled in the study (Fig. 1). Patients were then randomly allocated to one of four groups according to the prescribed therapy: amitriptyline $25 \mathrm{mg}$; amitriptyline $25 \mathrm{mg}$ and CBT; placebo and CBT; and placebo only (control). The use of placebo in controlled studies has been suggested as the most appropriate form to prove or disprove a given treatment modality when no universal methods of management are available so far.

Randomization was stratified by participant pain severity (as measured by the visual analog scale - VAS) to assure that the groups would be nearly balanced on these characteristics (Fig. 1). The randomization scheme was generated using the web site "www.randomization. com' and the researcher was blind to group distribution. Then, a different person was designated to allocate the patients in their groups, for the medicine distribution and to lead the patients to the CBT.

The most commonly cited reason for refusal to participate was the time commitment required to attend the sessions. During the study, 9 patients dropped out from the study and 1 was excluded from the study due to adverse events (self-report visual symptoms after taking the medicine).

\section{Research Protocol}

The protocol used in the present study was modified from Rizzatti-Barbosa et al. (1). Before randomization, participants were asked to discontinue, for 1 week, any treatment for TMD. All patients were

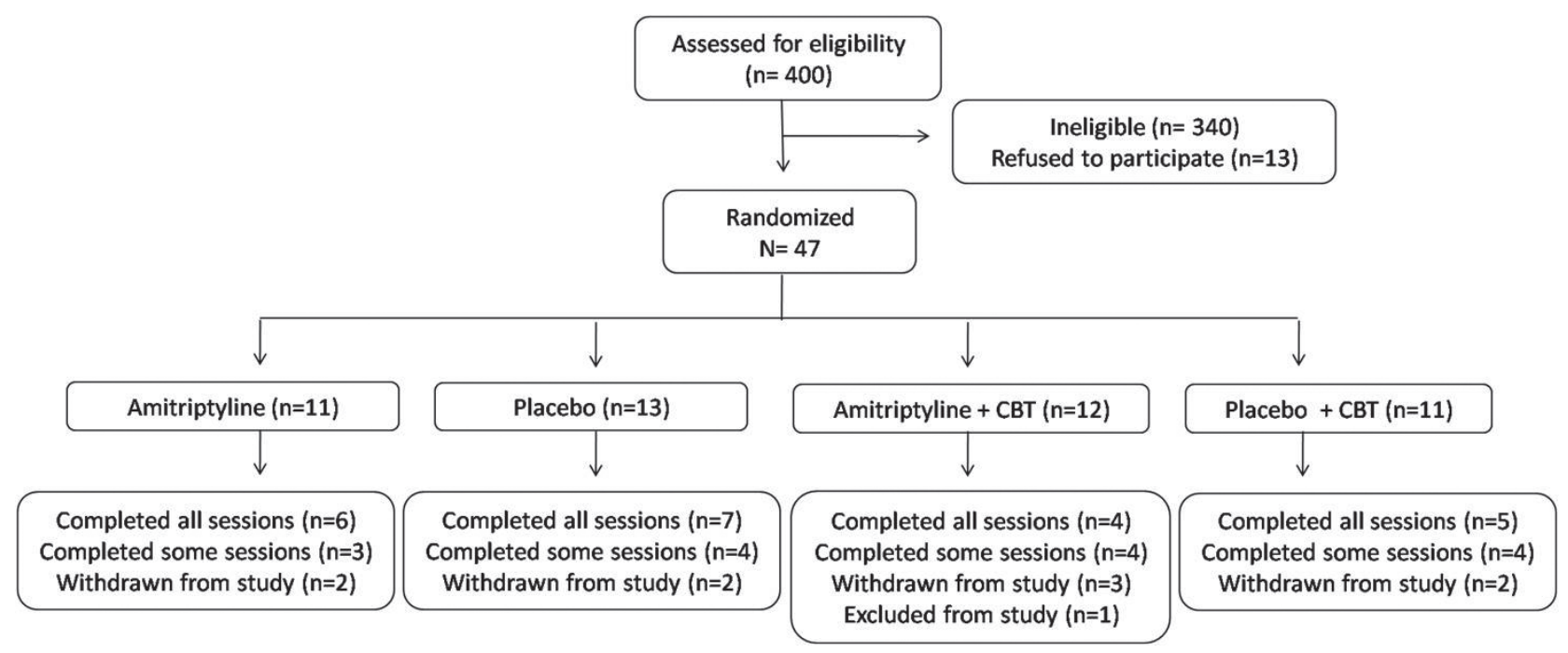

Figure 1. Flowchart illustrating the selection and distribution of the participants into the study groups. 
informed about the possible side-effects of amitriptyline. Those allocated into pharmacological treatment alone were instructed to take one tablet at bedtime for 7 consecutive weeks. For the groups that also received $\mathrm{CBT}$, the same protocol was used in combination with weekly 90 min CBT sessions for 7 weeks, conducted by $\mathrm{PhD}$-level psychologists, experienced in treating patients with pain. For this activity, participants were randomly allocated in groups of 4 participants on average. Sessions included education (expository lectures), relaxation training, and explanation of coping strategies, based on the CBT techniques.

\section{Assessment}

Prospective follow up occurred at three moments after the beginning of treatment: 1) 1 week; 2) 7 weeks, i.e. at the end of the treatment phase; and 3) 11 weeks.

A $100 \mathrm{~mm}$ visual analog scale (VAS) was used to evaluate the severity of pain. The 21-item Beck Depression Inventory (BDI) (3) was use to assess levels of depression; according to the BDI higher scores mean higher levels of depression. The 30-item Oral Health Impact Profile modified for orofacial pain

Table 1. Procedures performed at each evaluation time.

\begin{tabular}{cccc}
\hline Baseline & 1st week & 7th week & 11th week \\
\hline RDC/TMD & & VAS & VAS \\
VAS & & BDI & BDI \\
BDI & VAS & OHIP & OHIP \\
OHIP & & PSQI & PSQI \\
PSQI & & & \\
\hline
\end{tabular}

RDC/TMD: Research Diagnostic Criteria For Temporomandibular Disorders; VAS: Visual Analog Scale; BDI: Beck Depression Inventory; OHIP: Oral Health Impact Profile; PSQI: Pittsburg Sleep Quality Index. patients (OHIP) (4) was used to evaluate the quality of life; according to this inventory, higher scores indicates reduced quality of life. The 19-item Pittsburg Sleep Quality Index (PSQI) (5) was used to estimate the sleep quality; for this inventory higher scores suggests poor sleep quality. Table 1 shows the baseline and follow up procedures for this research.

During the research, the patients did not receive any other care for TMD as occlusal splints or physical therapy exercises.

\section{Statistical Analysis}

In order to assess the efficacy of the randomization process, the baseline characteristics of the 4 study groups on age, duration of pain and pain intensity were compared using two-way ANOVA.

Two-way ANOVA and Tukey's post-hoc test were used to investigate significant differences among the groups during the different follow-up sessions (pain intensity - VAS, levels of depression, quality of life and quality of sleep). These analyses considered all the patients that attended each session, i.e. 37 patients at the first follow up, 32 patients at the second, and 29 patients at the final follow up.

Univariate repeated measures ANOVA were used to investigate significant differences in each group during the research (pain intensity - VAS, levels of depression, quality of life and quality of sleep). These analyses considered just the patients that completed all sessions, i.e. 22 patients. The post-hoc calculation of power indicated a mean power for VAS scale for detecting difference in medication $=17 \%$ and for $\mathrm{CBT}=23 \%$.

The statistical analyses were performed using the software Statistics for Windows version 5.1. A level of significance of $5 \%$ was considered.

\section{RESULTS}

\section{Baseline Scores}

Table 2. Individuals' age (in years), pain duration (in months) and pain intensity (VAS) mean values and standard deviation (SD).

\begin{tabular}{lcccc}
\hline Measure & AMP & $\begin{array}{c}\text { AMP and } \\
\text { CBT }\end{array}$ & $\begin{array}{c}\text { Placebo and } \\
\text { CBT }\end{array}$ & $\begin{array}{c}\text { Placebo only } \\
\text { (control) }\end{array}$ \\
\hline Age & $32.4(9.5)$ & $36.7(13.6)$ & $38.2(11.1)$ & $34.5(11.0)$ \\
Pain duration & $84.5(64.2)$ & $70.2(102.9)$ & $92.0(86.3)$ & $54.5(47.9)$ \\
Pain intensity & $76.4(15.9)$ & $76.3(23.0)$ & $75.5(14.8)$ & $77.8(12.8)$ \\
\hline
\end{tabular}

$\mathrm{AMP}=$ amitriptyline; $\mathrm{CBT}$ : cognitive-behavioral therapy.

\section{Baseline Scores}

The mean age for the entire sample was 35.6 years (range 17-52 years). The mean pain duration was 72.35 months (range 6-384 months) and the $\mathrm{mm}$ (range 42-100 $\mathrm{mm}$ ).

The mean age, as well mean pain intensity was 76.5 
as baseline duration and intensity of pain, for each group, are shown on Table 2. There were no statistically significant differences among the four groups for any of these measures.

\section{Treatment Effectiveness}

The VAS, BDI, OHIP and PSQI scores at baseline and at the follow-up visits are presented in Table 3.

There were no significant differences in the VAS scores of the four groups at baseline and 11 weeks after the beginning of treatment $(p>0.05)$. One week after the beginning of treatment, those receiving amitriptyline and CBT and those receiving only placebo and CBT had VAS scores significantly higher than the other groups $(\mathrm{p}=0.03)$. In the 7th week, the amitriptyline and CBT group had significantly higher VAS scores than the amitriptyline group $(\mathrm{p}=0.01)$.

The VAS scores decreased significantly in the 1st week, compared with the baseline for the amitriptyline and CBT group and the placebo and CBT group $(\mathrm{p}<0.05)$. Significant reductions were seen at the 7th week, compared with baseline, for the four groups $(p<0.05)$, and at the 11th week, compared with baseline, for the amitriptyline group and amitriptyline and CBT group.

The BDI scores of the four groups were not

Table 3. Visual Analog Scale (VAS), Beck Depression Inventory (BDI), Oral Health Impact Profile (OHIP) and Pittsburg Sleep Quality Index (PSQI) mean scores for each group.

\begin{tabular}{lccccc}
\hline \multirow{2}{*}{ Measure } & $\begin{array}{c}\text { Evaluation } \\
\text { time }\end{array}$ & AMP & $\begin{array}{c}\text { AMP and } \\
\text { CBT }\end{array}$ & $\begin{array}{c}\text { Placebo and } \\
\text { CBT }\end{array}$ & $\begin{array}{c}\text { Placebo } \\
\text { only (control) }\end{array}$ \\
\hline \multirow{4}{*}{ VAS } & Baseline & 76.4 & 76.3 & 75.5 & 77.8 \\
& 1st wk & 38.4 & 63.2 & 54.6 & 41.0 \\
& 7th wk & 24.8 & 49.1 & 20.8 & 42.7 \\
& 11th wk & 26.7 & 39.0 & 38.7 & 52.6 \\
BDI & Baseline & 13.6 & 18.4 & 14.3 & 21.9 \\
& 7th wk & 7.1 & 9.5 & 2.0 & 15.7 \\
& 11th wk & 11.3 & 7.4 & 6.0 & 11.2 \\
& & & & & \\
OHIP & Baseline & 13.4 & 11.1 & 11.4 & 11.1 \\
& 7th wk & 4.8 & 2.8 & 6.7 & 8.5 \\
& 11th wk & 6.4 & 7.0 & 5.4 & 9.7 \\
& & & & & \\
& BSQI & 7.0 & 9.7 & 8.4 & 9.0 \\
& 7th wk & 5.4 & 10.1 & 6.7 & 9.4 \\
\hline
\end{tabular}

$\mathrm{AMP}=$ amitriptyline $\mathrm{CBT}=$ cognitive-behavioral therapy. different at baseline or at 7 weeks after the beginning of the treatment $(p>0.05)$. Eleven weeks after the beginning of the treatment, the placebo and CBT group had significantly lower BDI scores than the placebo only (control) group $(\mathrm{p}=0.02)$. The BDI scores were barely significant at the 7th week, compared with baseline, for the placebo and CBT group $(\mathrm{p}=0.03)$.

The OHIP scores of the four groups did not vary significantly from each other during the research $(p>0.05)$. The OHIP score were significantly different only at the 7th week, compared with baseline, for the placebo and CBT group $(\mathrm{p}=0.01)$.

The PSQI scores for the four groups were not significantly different, at either baseline or at the other evaluation times $(\mathrm{p}>0.05)$.

\section{DISCUSSION}

The aim of this study was to evaluate the efficacy of CBT and amitriptyline, in combination or alone, on the intensity of pain, levels of depression, quality of life and quality of sleep in patients with TMD. Comparing the groups of this research, the results showed that no significant differences were detected between groups for the pain and depression levels, quality of life and sleep.

It has been stated that TMD pain is episodic and self-limiting in most of the cases (6). It means that levels of pain can fluctuate over time. As a consequence, if someone seeks treatment when their level of pain is at its maximum (as the patients of the present study, who exhibited VAS value of $76.5 \mathrm{~mm}$ of pain intensity, in average), then pain can be expected to decrease towards its characteristic level whether or not treatment is given (7). This phenomenon is known as regression to the mean. Whitney and Von Korff (7) stated that when patient seeks treatment for pain conditions, subsequent improvement may be due to three types of effects: specific effect of treatment, placebo effect or, as stated above, regression to mean (within-subject variation across 
time). This is an important statement when judging the present results.

All four groups had improvements during the present study, although sometimes the differences were not statistically significant. There was a decrease of $55 \%$, on average, in pain intensity. This finding is comparable to those of Litt et al. (8), who stated that patients with TMD reported a $40 \%$ decrease in pain after standard treatment combined or not with CBT.

Considering the use of CBT for TMD treatment, the published outcomes differ broadly, and this might be explained by differences in the methodologies or the absence of control groups in some studies. This statement was confirmed by Liu et al. (9) in a systematic review about the effectiveness of the CBT for patients with TMD. Those authors could include only five studies with some methodological limitations and concluded that there is a clear and urgent need for well-designed randomized controlled trials examining CBT for TMD. To minimize this problem, Orlando et al. (10) suggested that strong efforts should be directed toward the identification of standardized treatment protocols, which are necessary to allow cross-center comparisons and replication of studies.

Physical, cognitive, affective and behavioral factors are all associated, and contribute to the experience of chronic pain. Astin (11) affirmed that while the nociceptive experience may remain unchanged, the emotional and cognitive components of the pain experience may be significantly diminished, resulting in less suffering. This may explain the effect of CBT on pain intensity. Dworkin et al. (12) found comparable results when comparing usual conservative treatment versus 6 sessions of $\mathrm{CBT}$ in combination with usual conservative treatment, during 1 year of follow up. Those authors stated that the overall improvement in both groups might be due to either efficacy of usual treatment, or to the natural cyclic course of TMD. Moreover, Orlando et al. (10) stated that the effectiveness of CBT in improving pain ratings did not appear to be related to the treatment itself, but rather associated with spontaneous pain remission.

Table 3 shows that, 1 month after the end of treatment, the amitriptyline and CBT group and the placebo and CBT group presented BDI scores that were lower than 11 , indicating minimum levels of depression. This finding suggests that CBT was effective in the control of depression, although the use of such therapy was not primarily devoted to treat depressive symptoms.
It can be observed that depression was not a primary inclusion criterion and was observed only in $38.3 \%$ of patients at baseline.

When the use of TCAs is considered, Plesh et al. (13) reported $50 \%$ improvement in pain intensity when using $30 \mathrm{mg}$ of amitriptyline, which is comparable with our findings. Furthermore, the amitriptyline and CBT group was the only group in which patients continued to improve according to the VAS results when treatment was discontinued, while all other groups worsened in the same period. Final figures for these groups, however, were maintained lower than those observed at baseline. This is supported by the results of Dworkin et al. (14), who stated and found that CBT interventions followed by usual treatment for TMD were associated with longterm decreases in pain intensity.

The analgesic mechanisms of TCAs are unclear. It has been speculated that low doses of amitriptyline, among others effects, stimulate descending serotonergic and noradrenergic antinociceptive pathways, which in turn influence endogenous opioids (15). It is also assumed that no antidepressant action is achieved with this dosage, although some improvement in humor and/ or sleep patterns can also explain the improvements obtained in chronic patients exposed to long-term use of such medications. Dosages were kept in $25 \mathrm{mg}$ and not titrated in the present investigation. Higher doses could lead to a more evident difference among groups, and should be considered in future studies.

Alternatively, higher doses may induce more severe adverse effects. McQuay, Carroll and Glynn (16) found that the analgesic efficacy was greater for the highest dose of amitriptyline ( $75 \mathrm{mg}$ compared with 50 and $25 \mathrm{mg}$ ); however some patients using $75 \mathrm{mg}$ and 50 $\mathrm{mg}$ dropped out from the study because of adverse events. Therefore the initial dose of amitriptyline should be low, and when the analgesic effect is insufficient and adverse effects are not limiting, increments may be made (16).

The placebo effect can also be part of this scenario and is defined as the positive physiological and psychological changes associated with the administration of inert substances or procedures (17). Substantial evidence implicates the endogenous opioid system in the mediation of the placebo effect under conditions of expected analgesia (17). Sharav et al. (18) did not find differences between the control group and the group that received $25 \mathrm{mg}$ of amitriptyline, after 1 week of the beginning of treatment. Authors concluded that the placebo effect on pain is high at the early periods 
of drug administration. The use of placebo in controlled studies has been suggested as the most appropriate form to prove or disprove a given treatment modality, especially in the case of multifactorial entities, when no universal methods of management are available so far. The management of chronic TMD, as studied in the present investigation, clearly illustrates such situation. Table 3 shows that, according to our data, after the first follow up visit, the control group did not improve any longer.

Greene and Laskin (19) stated that those individuals who respond favorably to a treatment will generally continue to feel comfortable and function well over long periods of time, regardless of whether the treatment was real or sham. This was observed in the present investigation, where the improvement achieved by the control group (when compared with initial VAS figures) was maintained after the end of drug administration.

Considering the quality of life, Turp et al. (20), in a systematic review about the influence of TMD interventions influence in oral health-related quality of life found that, except for the patients with multiple TMJ surgeries, all forms of interventions led to at least some improvement on the quality of life. Their findings are in agreement with the present results, which showed a slight, but not statistically significant, improvement in OHIP score.

The quality of sleep appeared to be slightly improved at the end of investigation for the groups that received amitriptyline, but this improvement was not statistically significant. This may be due to the sedative effect of this drug, since decreased sleep may be associated with increased perception of pain; if so, sedation may be advantageous (21). In addition, CBT may also produce improvements in sleep. This improvement may not be associated with complete relieve of pain, but it would contribute to improve quality of life.

The present pilot study has certain limitations that reduce the strength of our conclusions. Using the VAS scale for sample size calculation, based on the mean within variability of the groups $(n=21)$, to detect a difference of 10 units in the VAS scale, using $p=0.05$ and power $=0.80$, is necessary a sample size of 36 subjects in each group. The small sample size seems to be the major limitation. Moreover, the dropouts during the research may have occurred due to the time commitment required to attend the sessions and also because of the financial situation of some patients, who might have experimented difficulties to afford transportation to the clinic facilities. In a future study, we might consider the possibility of reimbursing the transportation expenses, although we are aware that this may influence patients to demonstrate positive outcome.

Future studies using the same methodology and larger sample sizes will be done to establish if the absence of statistical differences found in the present pilot study, for some variables, are due to the small sample size or to the natural cyclic course of TMD. Even with the limitations, the results of this pilot study suggest that all the interventions may be effective for chronic patients with TMD. Moreover, the combination between amitriptyline and CBT seems to produce better results, even after the end of treatment.

A fundamental requirement for improved therapeutic outcomes is the clinician's acceptance of the importance of psychological factors and knowledge of the efficacy and effectiveness of psychological interventions (22). Clinicians should consider the establishment of a multidisciplinary team, consisting of a dentist and a psychologist and/or physician to provide treatment for chronic patients with TMD. As suggested by Turp et al. (22) in a systematic review that evaluated the superiority of multimodal as opposed to simple therapy in patients with TMD and found that in patients with TMD with psychological disturbances, patients benefited more from a combined therapeutic approach compared with simple care.

The results of the present study suggest that the association between amitriptyline and CBT may be effective in reducing pain and depression levels, as well as in improving the quality of life and sleep in chronic patients with TMD.

\section{RESUMO}

O objetivo deste estudo piloto foi avaliar a eficácia do uso de uma terapia cognitivo comportamental (TCC) e da amitriptilina, um antidepressivo tricíclico, no tratamento de pacientes portadores de disfunções temporomandibulares (DTM) crônicas. Quarenta e sete mulheres (com 35,4 anos em média) diagnosticadas com DTM crônica foram selecionadas e divididas em 4 grupos: amitriptilina; amitriptilina + TCC; placebo + TCC; e placebo (controle) e controle. As pacientes foram submetidas às terapias por 7 semanas consecutivas. As reavaliações aconteceram 1, 7e 11 semanas após o início da terapia (11 semanas de avaliações). Foram avaliados a presença e severidade de dor, o nível de depressão, a qualidade de vida e do sono. Os dados foram avaliados através de ANOVA, Qui-quadrado e teste de Cochran, considerando um nível de significância de 5\%. Foram observadas melhoras 
significantes em todos os fatores avaliados para todos os grupos, porém não foram observadas diferenças entre os grupos. Após o final da terapia, os resultados positivos persistiram apenas para o grupo amitriptilina + TCC. Os resultados sugerem que o uso associado de amitriptilina e TCC no tratamento de pacientes portadores de DTM crônicas pode ser efetivo na melhora da dor, nível de depressão, qualidade de vida e do sono.

\section{ACKNOWLEDGEMENTS}

The authors want to thank all the study participants for their cooperation and CAPES - Brazil for the financial support.

\section{REFERENCES}

1. Rizzatti-Barbosa CM, Nogueira MT, de Andrade ED, Ambrosano GM, de Barbosa JR. Clinical evaluation of amitriptyline for the control of chronic pain caused by temporomandibular joint disorders. Cranio 2003;21:221-225.

2. Dworkin SF, LeResche L. Research diagnostic criteria for temporomandibular disorders: review, criteria, examinations and specifications, critique. J Craniomandib Disord 1992;6:301-355.

3. Beck AT, Ward CH, Mendelson M, Mock J, Erbaugh J. An inventory for measuring depression. Arch Gen Psychiatry 1961;4:561-571.

4. Murray H, Locker D, Mock D, Tenenbaum HC. Pain and the quality of life in patients referred to a craniofacial pain unit. J Orofac Pain 1996;10:316-323.

5. Buysse DJ, Reynolds CF 3rd, Monk TH, Berman SR, Kupfer DJ. The Pittsburgh Sleep Quality Index: a new instrument for psychiatric practice and research. Psychiatry Res 1989;28:193213.

6. Von Korff M, Dworkin SF, Le Resche L, Kruger A. An epidemiologic comparison of pain complaints. Pain 1988;32:173183.

7. Whitney CW, Von Korff M. Regression to the mean in treated versus untreated chronic pain. Pain 1992;50:281-285.

8. Litt MD, Shafer DM, Kreutzer DL. Brief cognitive-behavioral treatment for TMD pain: long-term outcomes and moderators of treatment. Pain 2010;151:110-116.

9. Liu HX, Liang QJ, Xiao P, Jiao HX, Gao Y, Ahmetjiang A. The effectiveness of cognitive-behavioural therapy for temporomandibular disorders: a systematic review. J Oral Rehabil 2011 [Epub ahead of print. DOI: 10.1111/j.13652842.2011.02239.x.].
10. Orlando B, Manfredini D, Salvetti G, Bosco M. Evaluation of the effectiveness of biobehavioral therapy in the treatment of temporomandibular disorders: a literature review. Behav Med 2007;33:101-118.

11. Astin JA. Mind-body therapies for the management of pain. Clin J Pain 2004;20:27-32.

12. Dworkin SF, Turner JA, Mancl L, Wilson L, Massoth D, Huggins $\mathrm{KH}$, et al.. A randomized clinical trial of a tailored comprehensive care treatment program for temporomandibular disorders. J Orofac Pain 2002;16:259-276.

13. Plesh O, Curtis D, Levine J, McCall WD Jr. Amitriptyline treatment of chronic pain in patients with temporomandibular disorders. J Oral Rehabil 2000;27:834-841.

14. Dworkin SF, Turner JA, Wilson L, Massoth D, Whitney C, Huggins $\mathrm{KH}$, et al.. Brief group cognitive-behavioral intervention for temporomandibular disorders. Pain 1994;59:175-187.

15. Brown RS, Bottomley WK. Utilization and mechanism of action of tricyclic antidepressants in the treatment of chronic facial pain: a review of the literature. Anesth Prog 1990;37:223-229.

16. McQuay HJ, Carroll D, Glynn CJ. Dose-response for analgesic effect of amitriptyline in chronic pain. Anaesth 1993;48:281-285.

17. Zubieta JK, Bueller JA, Jackson LR, Scott DJ, Xu Y, Koeppe RA, et al.. Placebo effects mediated by endogenous opioid activity on mu-opioid receptors. J Neurosci 2005;25:7754-7762.

18. Sharav Y, Singer E, Schmidt E, Dionne RA, Dubner R. The analgesic effect of amitriptyline on chronic facial pain. Pain 1987;31:199-209.

19. Greene CS, Laskin DM. Long-term evaluation of conservative treatment for myofascial pain-dysfunction syndrome. J Am Dent Assoc 1974;89:1365-1368.

20. Turp JC, Motschall E, Schindler HJ, Heydecke G. In patients with temporomandibular disorders, do particular interventions influence oral health-related quality of life? A qualitative systematic review of the literature. Clin Oral Implants Res 2007;18:127-137.

21. Kreisberg MK. Tricyclic antidepressants: analgesic effect and indications in orofacial pain. J Craniomandib Disord 1988;2:171177.

22. Turp JC, Jokstad A, Motschall E, Schindler HJ, Windecker-Getaz I, Ettlin DA. Is there a superiority of multimodal as opposed to simple therapy in patients with temporomandibular disorders? A qualitative systematic review of the literature. Clin Oral Implants Res 2007;18Suppl3:138-150. 